\title{
Egr-1 mRNA Expression Is a Marker for the Direction of Mammalian Ocular Growth
}

\author{
Regan S. Ashby, ${ }^{1-3}$ Guang Zeng, ${ }^{1,4}$ Amelia J. Leotta, ${ }^{1}$ Dennis Y. Tse, ${ }^{1,5}$ and Sally A. McFadden ${ }^{1}$ \\ ${ }^{1}$ Vision Sciences Group, School of Psychology, Faculty of Science and IT, The University of Newcastle, New South Wales, Australia \\ ${ }^{2}$ Faculty of Education, Science, Technology and Mathematics, University of Canberra, Canberra, Australian Capital Territory, \\ Australia \\ ${ }^{3}$ ARC Centre of Excellence in Vision Science, Research School of Biology, Australian National University, Canberra, Australian \\ Capital Territory, Australia \\ ${ }^{4}$ Department of Ophthalmology, Daqing Oilfield General Hospital, Saertu, Daqing, China \\ ${ }^{5}$ School of Optometry, The Hong Kong Polytechnic University, Hung Hom, Kowloon, Hong Kong
}

Correspondence: Regan S. Ashby, Faculty of Education, Science, Technology and Mathematics, University of Canberra, Canberra, ACT 2601, Australia;

regan.ashby@canberra.edu.au.

Submitted: January 22, 2013

Accepted: June 28, 2014

Citation: Ashby RS, Zeng G, Leotta AJ, Tse DY, McFadden SA. Egr-1 mRNA expression is a marker for the direction of mammalian ocular growth. Invest Ophthalmol Vis Sci. 2014;55:5911-5921. DOI:10.1167/ iovs.13-11708
Purpose. The immediate early gene Egr-1 is thought to form part of the pathway that mediates abnormal ocular growth. This study investigated whether the mRNA expression levels of $E g r-$ 1 in a mammalian retina are modulated differentially, depending on the direction of ocular growth.

Methons. To induce accelerated growth and myopia, guinea pigs wore a -5 diopter (D) lens over one eye from 4 to 11 days of age. To induce inhibited growth, the lens was removed after 7 days of $-5 \mathrm{D}$ lens wear, and the eye allowed to recover from myopia for 3 days. Ocular parameters and Egr-1 mRNA levels were subsequently assessed, and compared to untreated fellow eyes and eyes from untreated littermates. Possible circadian changes in Egr-1 mRNA levels were also determined in 18 additional animals by taking measures every 4 hours during a 24-hour cycle.

Results. Ocular compensation to a $-5 \mathrm{D}$ lens occurred after 7 days $(\Delta-4.8 \mathrm{D}, \Delta+147 \mu \mathrm{m}$ growth, $N=20)$. In 5 highly myopic eyes $(\Delta-7.4 \mathrm{D}), E g r-1$ mRNA levels in the retina were significantly downregulated relative to contralateral control (51\%) and age-matched untreated (47\%) eyes. Three days after the $-5 \mathrm{D}$ lens was removed, eyes had recovered from the myopia $(\Delta-0.5 \mathrm{D}$, relative change of $+2.9 \mathrm{D}, N=4)$ and $E g r-1 \mathrm{mRNA}$ levels were significantly elevated relative to contralateral (212\%) and untreated (234\%) eyes, respectively. Normal Egr1 mRNA expression was higher in the middle of the day than in the middle of the night. Immunolabeling showed strong Egr-1 reactivity in cell bodies in the inner nuclear and ganglion cell layers.

Conclusions. Egr-1 mRNA levels in a mammalian retina show a bi-directional persistent response to opposing ocular growth stimuli. This suggests retinal Egr-1 might act as a signal for the direction of ocular growth in different species.

Keywords: myopia, Egr-1, guinea pig, retina, refractive error
M yopia is an ocular disorder brought about by a mismatch between the axial length of the eye and its optical properties, and commonly arises from excessive ocular elongation during development. Research into possible optical and pharmacologic treatments for the prevention of myopia has been aided by the discovery that the rate of eye growth can be manipulated experimentally in animals by altering their visual experience during development. ${ }^{1,2}$ Myopia can be reliably induced in young growing eyes by either form deprivation ${ }^{3-7}$ (FDM) or by imposing hyperopic defocus with a negative lens $^{8-13}$ (lens-induced myopia, LIM), both of which induce excessive elongation of the eye. In humans, degradation of the retinal image during ocular development through drooping eyelids (ptosis) ${ }^{14}$ or cataracts ${ }^{15}$ may also result in myopia. In contrast, inhibition of ocular growth occurs when the eye is allowed to recover from the myopia induced during FDM or LIM, by the subsequent removal of the diffuser or negative lens, ${ }^{3,7}$ and when young animals wear a positive lens. ${ }^{8-11,13,16}$
Eye growth is thought to be regulated by retinal mechanisms that act locally within the eye, producing a cascade of changes that ultimately affect the structure of the sclera. Evidence for the role of the retina in the control of eye growth comes from studies involving optic nerve section ${ }^{17,18}$ (Wildsoet CF, et al. IOVS 2010;51:ARVO E-Abstract 1737) or the pharmacologic blockade of action potentials in the optic nerve. ${ }^{19,20}$ The results of these studies indicate that gross regulation of ocular growth appears not to require innervation from the brain. Furthermore, partial form deprivation induces myopic changes only in the corresponding sector of the eye, a process difficult to explain by a global centrally driven pathway ${ }^{7}$ (McFadden SA. IOVS 2002; 43:ARVO E-Abstract 189).

A retinal factor implicated in the control of eye growth is the immediate early gene known as early growth response-1 (Egr-1). Egr-1 is a transcription factor that encodes a short-lived nuclear protein with a zinc finger-binding domain. Its expression is normally rapidly and transiently induced by 
extracellular stimuli. However, alterations in Egr-1 expression in response to specific stimuli can be delayed by hours to days, with its expression remaining altered for prolonged periods. ${ }^{21}$ Egr-1 was first implicated in the modulation of eye growth by the observation in chick retina that glucagonergicimmunoreactive amacrine cells, positively labeled for the Egr1 peptide, show a bidirectional response to opposing growth stimuli. Specifically, the percentage of these cells that expresses both glucagon and Egr-1, relative to those expressing glucagon alone, decreases within 2 hours of FDM or LIM, and increases within 2 hours of positive lens wear or removal of diffusers from previously form-deprived eyes. ${ }^{22}$ Additionally, in the avian retina, $E g r-1$ mRNA levels are rapidly downregulated in response to FDM or LIM, and remain downregulated during the entire period of increased ocular growth. $^{23}$ Following diffuser removal, Egr-1 mRNA levels rapidly rise above that seen in form-deprived and control untreated chick eyes. ${ }^{24}$ In response to positive lenses (which cause growth suppression), earlier work by Simon et al. ${ }^{25}$ have indicated that Egr-1 mRNA levels are initially downregulated after 15 minutes of $+7 \mathrm{D}$ lens wear, but after 30 minutes revert to a significant upregulation. Therefore, Egr-1 mRNA levels generally show a bidirectional response in chicks. That is, opposite changes occur in Egr-1 expression depending on the direction of ocular growth: increased expression with growth inhibition and decreased expression with growth acceleration. The ability of pharmacologic agents, which block the development of experimental myopia, to prevent the downregulation in $E g r-1$ mRNA expression associated with $\mathrm{FDM}^{24}$ and $\mathrm{LIM}^{23}$ in chicks suggests that decreases in Egr1 expression may be an obligatory precursor to myopic ocular growth in the avian eye.

Evidence also suggests an association between changes in ocular growth rates and alterations in $E g r-1$ expression in mice. Egr-1 knockout mice show a relative myopic shift compared to wild-type control animals. ${ }^{26}$ Egr-1 mRNA levels are also reduced after 1 hour of diffuser wear in mice. ${ }^{27}$ However, Egr-1 mRNA levels have not been directly measured in other mammals or primates, or during lens wear, and it is unknown whether upregulation in Egr-1 mRNA levels might occur during inhibited ocular growth in species other than chicks.

In rhesus monkeys, the number of amacrine cells immunoreactive (IR) for Egr-1 is altered in response to visual manipulation. Specifically, Egr-1 IR cell numbers decrease after a brief period of diffuser wear $(N=3)$ and apparently increase after a brief period of positive lens wear $(N=3) \cdot{ }^{28}$ These changes are relative to fellow eyes wearing a plano lens, but the mean number of cells IR for Egr-1 in the plano-wearing fellow eyes differs significantly between these two groups. In particular, Egr-1 cell numbers are significantly lower in the plano eyes of animals wearing a positive lens, suggesting the lens effect is instead manifested in the fellow eye. ${ }^{28}$ The authors have proposed that this unexpected result may have been due to the monkeys using the positive lens as a substitute for accommodation. The consequences of this would be that the positive lens-wearing eye would experience clear vision, while the yoked fellow eye wearing a plano lens would experience hyperopic defocus. ${ }^{28}$ If true, then the conclusion from this monkey experiment is that Egr-1 immunoreactivity decreases in the eyes experiencing growth from hyperopic defocus or form deprivation, but the issue of a bidirectional change in which Egr-1 is elevated with inhibited growth remains unresolved.

The retinal cells that changed their immunoreactivity for Egr-1 peptide in the rhesus monkey are a subpopulation of gamma-aminobutyric acid (GABA) expressing amacrine cells and a subpopulation of ON-bipolar cells. ${ }^{28}$ In chicken retina, Egr-1 immunoreactivity has been reported to be associated with short periods of defocus in glucagonergic amacrine cells, but such cells have not been detected in mouse ${ }^{29}$ or monkey retina. $^{28}$ This suggests that species differences occur in the localization of retinal Egr-1.

In the current study, we sought to directly measure Egr-1 mRNA transcript levels by using semi-quantitative real-time reverse transcription polymerase chain reaction (qRT-PCR) in a mammalian retina after animals experienced either positive or negative defocus to induce opposite changes in ocular growth. Specifically, changes in $E g r-1$ mRNA levels were measured in retinas from young guinea pigs that had worn a negative lens for a week (which induced myopic growth in response to hyperopic defocus) or where the negative lens was worn for a week and then removed for several days (which causes recovery from myopia and inhibited ocular growth). In our study, we used time points at which compensation for the opposing growth responses were well advanced to avoid any initial transient changes associated with subtle changes in light or contrast, and to determine if longer-term changes in Egr-1 gene expression occur. Additionally, using immunohistochemistry, we determined the location of cells expressing Egr-1 in the guinea pig retina.

\section{Materials ANd Methods}

\section{Animals and Housing}

Approximately 50 guinea pigs (Cavia porcellus, pigmented, tricolored) were bred at the University of Newcastle, New South Wales, Australia, and housed with their mothers and littermates in opaque plastic boxes $(65 \times 45 \times 20 \mathrm{~cm})$ with stainless wire lids. Lighting was provided by three white lightemitting diodes (1400 mA, Luxeon III Star; Philips Lumileds Lighting Company, USA) evenly diffused through a Perspex barrier located $20 \mathrm{~cm}$ above each box. The luminance in the center of each holding box was 500 lux, and lights were on a 12/12 hour light/dark cycle (lights on at 10 AM). All procedures were approved by the University of Newcastle Animal Experimentation Ethics Committee and conformed to the ARVO Statement for the Use of Animals in Ophthalmic and Vision Research.

\section{Experimental Treatments}

Experiment 1: Measurement of Egr-1 Expression in Retinas With Myopia or Inhibited Myopia. Animals within a litter were randomly assigned to one of three groups: myopia $(N=5)$; myopia recovery $(N=4)$; or normal untreated animals $(N=5)$. Myopia was induced in the first two groups with a -5 diopter (D) lens worn in front of one eye for 7 days (from 4-11 days of age). In the myopia group, the retinas of both eyes were extracted at 11 days of age. In the myopia recovery group, the $-5 \mathrm{D}$ lens was removed at 11 days of age, and the eye recovered from the induced myopia for the next 3 days, followed by retinal extraction at 14 days of age. In the third group, retinas were extracted at 11 days of age in normal animals that did not wear a lens, to ascertain the normal baseline. Refractive error was measured at $\mathbf{1 0}$ days of age in the myopia and normal groups, or at both 10 and 13 days of age in the myopia recovery group. Refractive error measurements were undertaken the day before retinal extraction to ensure no measurement factors interfered with the retinal state immediately before extraction. Lenses were initially applied or removed 6 hours after lights were switched on (at 4 PM) and retinal extractions were also done at 4 PM (i.e., in the middle of their light cycle). Retinal tissue was analyzed for changes in Egr-1 expression by using qRT-PCR. 
TABLE 1. Gene-Specific RT-PCR Primer Information

\begin{tabular}{lccccc}
\hline Gene Product & GenBank Acc. & Forward Primer (5'-3') & Reverse Primer (5'-3') & Efficiency & Product Size, bp \\
\hline Egr-1 & - & GCACCCAACAGTGGCAACAC & ACTGCTGTCGTTGGATGGCA & 1.91 & 180 \\
$\beta$-Actin & NM_001172909.1 & CACCAGGGAGTCATGGTAGG & GGTGTGGTGCCAGATCTTCT & 1.86 & 150 \\
\hline
\end{tabular}

Experiment 2: Ocular Elongation After 7 Days of -5 D Lens Wear. To determine the amount that eyes grew with $a-5$ D lens under the same timing conditions as in experiment 1 , eleven additional guinea pigs were raised with $-5 \mathrm{D}$ lenses worn on one eye for 1 week from 4 days of age. At 10 days of age, refractive error was measured as described in experiment 1. Immediately after the refractive error measurements, animals were anesthetized with $1.5 \%$ isoflurane in oxygen and the ocular length measured by using high-frequency ultrasonography.

Experiment 3: Measurement of Diurnal/Circadian Rhythm in Egr-1 Expression. Eighteen guinea pigs were raised from 4 to 11 days of age. At 11 days of age, retinas were extracted at 1, 5, 9, 13, 17, and 21 hours after the start of the light cycle (six retinas per time point). Housing, lighting conditions, retinal extraction, and measurement of Egr-1 expression were identical to those in experiment 1. Egr-1 expression was normalized against the mean value for $\beta$-actin (reference control gene) at each time point.

Experiment 4: Immunohistochemical Localization of Egr-1 in the Guinea Pig Retina. Approximately 12 retinas were extracted from both adult and untreated 11-day-old guinea pigs raised under the same lighting conditions as in experiment 1. Egr-1 was localized in the extracted retinas using immunohistochemistry.

\section{Lenses and Application}

Concave $-5 \mathrm{D}$ polymethylmethacrylate lenses (diameter and optical zone of $14 \mathrm{~mm}$, base curve of $8.5 \mathrm{~mm}$; Gelflex, Perth, Australia) were worn in front of one eye. They were attached by using hook and loop fasteners (Birch Haberdashery and Craft, Melbourne, Australia) as previously described. ${ }^{11}$ The distance of the lens apex from the cornea $(d)$ was approximately $5 \mathrm{~mm}$; therefore, the effective power at the anterior corneal surface $\left(F_{e}\right)$ was $-4.88 \mathrm{D}\left(F_{e}=\frac{F}{1-(d \times F)}\right)$. Lenses were replaced with clean lenses daily, during which time the animals were kept in the dark.

\section{Ocular Measurements}

Refractive error was measured with white light steak retinoscopy 1 hour after cycloplegia had been induced (with 1-2 drops of cyclopentolate applied to the cornea for 1 minute). Measurements were taken in both the vertical and horizontal meridian and averaged to give spherical equivalent refractive error as previously described. ${ }^{30}$ In experiment 2 , internal ocular distances on axis were measured by using highfrequency ultrasonography (20 MHz probe; Panametrics Model 176599 controller [Olympus, Notting Hill, Vic, Australia] and $100 \mathrm{MHz}$ a/d Sonix 8100 sampling board [Springfield, VA, USA]) as previously described. ${ }^{31}$ Ocular length was defined as the distance from the front of the cornea to the back of the sclera.

\section{Retinal Extraction}

After deep anesthesia (injection of $130 \mathrm{mg} / \mathrm{kg}$ of pentobarbitone sodium into the heart in animals anesthetized with $1.5 \%$ isoflurane in oxygen) the eyes were rapidly enucleated (within approximately 1 minute to prevent degradation of the retinal tissue). The cornea was removed by a circumference cut at the limbus. The remaining posterior eye cup was floated in chilled phosphate-buffered saline (PBS: $137 \mathrm{mM} \mathrm{NaCl}, 2.7 \mathrm{mM} \mathrm{KCl}$, $11.3 \mathrm{mM} \mathrm{Na}_{2} \mathrm{HPO}_{4}, 1.5 \mathrm{mM} \mathrm{KH} \mathrm{PO}_{4}, \mathrm{pH}$ 7.4; Sigma-Aldrich, Sydney, Australia). The retina was separated from the underlying RPE and choroid by inserting a finely tipped spatula between the layers, allowing the retina to float to the surface of the PBS solution, free of RPE or choroid. In general, retinal tissue from light-adapted animals was readily collected free of RPE. If fragments of RPE remained, they were lightly brushed from the retina. For PCR analysis, each retina was placed in an Eppendorf tube (North Ryde, NSW, Australia) and immediately frozen by using liquid nitrogen and stored at $-80^{\circ} \mathrm{C}$ until analyzed. For immunohistochemistry, the vitreous was removed from the posterior eye cup and the whole eye cup was fixed by immersion in $4 \%$ paraformaldehyde in $0.1 \mathrm{M}$ phosphate buffer ( $\mathrm{pH} 7.4$ ) for 2 hours at $4^{\circ} \mathrm{C}$. Eye cups were washed once in PBS, cryoprotected in 30\% sucrose in PBS, embedded in O.C.T. compound (Tissue-Tek; Thermo Fisher Scientific, Inc., Scoresby, Vic, Australia), and quickly frozen on liquid nitrogen chilled ethanol (100\%) and stored at $-80^{\circ} \mathrm{C}$ until analyzed.

\section{Immunohistochemistry}

Fifteen-micrometer-thick vertical retinal sections were cut from the fixed embedded frozen eye cups using a cryostat (CM1850; Leica Microsystems, North Ryde, Australia). Sections were cut in the nasotemporal plane containing the optic nerve and were thaw mounted onto gelatin-coated glass slides and air-dried overnight. Sections were washed three times in PBS and incubated overnight at $4^{\circ} \mathrm{C}$ with rabbit monoclonal antibodies to Egr-1 (1:500, 15F7, No. 4153, Entrez-Gene ID No. 1958, SwissProt Acc. No. P18146; Cell Signaling Technology, Inc., Danvers, MA, USA). Slides were washed three times in PBS and then incubated in secondary antibody (1:500, donkey anti-rabbit secondary IgG, AffiniPure FITC 711-095-152; Jackson ImmunoResearch Lab, Inc., ABACUS, Qld, Australia) in a humidified chamber for 2 hours at room temperature. Negative controls were run and some sections were double-labeled for GABA (1:10,000, A0310; Sigma-Aldrich) or protein kinase C (1:100, PKC mouse monoclonal IgG, MC5; Santa Cruz Biotech, Dallas, TX, USA) and conjugated by secondary donkey anti-mouse IgG (1:300, AffiniPure Cy3 715-165-150).

\section{Real-Time Reverse Transcription-Polymerase Chain Reaction (qRT-PCR)}

The extraction of total RNA was undertaken by using a combined Trizol/Qiagen RNeasy (Qiagen Pty Ltd, Chadstone Centre, Vic, Australia) method as previously described. ${ }^{24,32}$ Purity of total RNA was checked by using gel electrophoresis and a bioanalyzer, and quantified by using a nanodrop spectrophotometer. Two micrograms of each sample were reverse-transcribed to first-strand cDNA by using a SuperScript VILO cDNA Synthesis Kit (Invitrogen, Mulgrave, Victoria, Australia). As Egr-1 has not been annotated in the guinea pig, primers were designed to detect conserved regions (high 
TABLE 2. Mean RE in Eyes Analyzed for Egr-1 (From Experiment 1), and Mean RE and Ocular Length Data From Experiment 2

\begin{tabular}{|c|c|c|c|c|c|}
\hline & \multirow[b]{2}{*}{ Eye } & \multicolumn{2}{|c|}{ RE Day 10, D } & \multicolumn{2}{|c|}{ RE Day $13, \mathrm{D}$} \\
\hline & & Mean & SEM & Mean & SEM \\
\hline \multicolumn{6}{|l|}{ Experiment 1} \\
\hline \multirow[t]{3}{*}{ Myopia, $N=5$} & Fellow & 3.72 & 0.48 & & \\
\hline & $-5 \mathrm{D}$ & -3.66 & 1.04 & & \\
\hline & Difference & -7.39 & 0.73 & & \\
\hline \multirow{3}{*}{$\begin{array}{l}\text { Myopia Recovery, } \\
\quad N=4\end{array}$} & Fellow & 4.05 & 1.29 & 3.58 & 0.31 \\
\hline & $-5 \mathrm{D}$ & 0.62 & 1.40 & 3.05 & 1.42 \\
\hline & Difference & -3.43 & 0.42 & -0.53 & 1.15 \\
\hline \multirow[t]{3}{*}{ Untreated, $N=5$} & Left & 4.12 & 0.18 & & \\
\hline & Right & 4.79 & 1.22 & & \\
\hline & Difference & 0.68 & 1.28 & & \\
\hline \multicolumn{4}{|l|}{ Experiment 2} & \multicolumn{2}{|c|}{ OL (MM) } \\
\hline \multirow[t]{3}{*}{ Myopia, $N=11$} & Fellow & 3.85 & 0.45 & 7.935 & 0.035 \\
\hline & $-5 \mathrm{D}$ & -0.34 & 0.87 & 8.083 & 0.064 \\
\hline & Difference & -4.19 & 0.62 & 0.147 & 0.048 \\
\hline
\end{tabular}

RE, refractive error; SEM, standard error of the mean; OL, ocular length.

homology) across rats (closest phylogenetic relative), mice, and humans (Table 1). Product specificity was validated through melt curve analysis, gel electrophoresis, and automated sequencing. Primer efficiency $(E)$ was determined for Egr-1 and the reference gene of $\beta$-actin ( $a c t b$, Sigma-Aldrich) from the slope of the curve generated through a cDNA dilution series, by using the formula $E=10^{(-1 / \text { slope })}$.

All reactions were performed on a RotorGene 3000 qRT-PCR cycler (Corbett Life Science [Qiagen], Chadstone Centre, Victoria, Australia). Cycling conditions included an initial denaturing phase of $95^{\circ} \mathrm{C}$ for 5 minutes, followed by 35 cycles of denaturation at $95^{\circ} \mathrm{C}$ for 25 seconds, annealing at $60^{\circ} \mathrm{C}$ for 15 seconds, and extension at $72^{\circ} \mathrm{C}$ for 20 seconds. Repeated takeoff values and sample amplification values obtained by the RotorGene v6.0 software were transferred into a custom-built Microsoft Excel (Microsoft Pty Ltd., North Ryde, NSW, Australia) spreadsheet.

\section{Sequencing of Egr-1 mRNA PCR Fragments}

For sequencing analysis, PCR products were purified, cleaned, and prepared by using a BigDye Terminator v3.1 Cycle Sequencing Kit, following the manufacturer's protocol (Applied Biosystems, Mulgrave, Victoria, Australia). All sequencing reactions were undertaken by a sequencing unit core facility (Biomedical Resource Facility at the Australian National University) by using $5 \mathrm{ng}$ of starting template and $3.2 \mathrm{pmol}$ of forward primer. Annotation of the PCR fragment was determined from the analysis of four separate retinal PCR samples, with each separate sample replicated/sequenced five times. Egr-1 sequence alignment and phylogenetic tree reconstruction from the sequence relationships were undertaken in Clustal W 2.1 (http://www.genome.jp/tools/clustalw/; provided in the public domain by Kyoto University Bioinformatics Center). Phylogenetic tree reconstruction was done against rat (NM_012551.2), mouse (NM_007913.5), human (NM_001964.2), chimpanzee (XM_517958.2), and rhesus monkey (XM_001107731.2).

\section{Statistical Analysis}

Results are presented as the mean \pm standard error of the mean. The difference in refractive error between the lens- wearing and fellow contralateral control eye was calculated for each animal and the mean difference is referred to as the "relative" myopia. For retinal Egr-1 mRNA levels, the mean normalized expression (MNE) of the target gene was calculated separately for each condition (treated, fellow contralateral control, and age-matched untreated control retinal tissue) as previously described. ${ }^{23,33}$ The MNE was calculated from the efficiency $(E)$ of the target gene to the power of its average threshold cycle (Ct) value $\left(E^{\mathrm{Ct}}\right.$, target), divided by the efficiency $(E)$ of the reference gene $(\beta$-actin) to the power of its average $\mathrm{Ct}$ value ( $E^{\mathrm{Ct}}$, reference). ${ }^{33}$ Statistical differences between the two eyes were based on matched pairs $t$-tests. Comparisons between treated, contralateral, and age- and littermatched untreated eyes were based on repeated measures oneway ANOVA followed by Holm-Sidak comparisons. Comparisons between different times in experiment 3 were based on one-way ANOVA followed by Holm-Sidak comparisons. Statistical analysis used JMP 7 (SAS, Cary, NC, USA) and SigmaPlotV11 (Systat Software, Inc., San Jose, CA, USA).

\section{Results}

\section{Changes in Refraction and Ocular Length}

Animals wearing -5 D lenses for 7 days in experiments 1 and 2 $(N=20)$ developed $-4.84 \pm 0.62 \mathrm{D}$ of relative myopia $\left(t_{19}=\right.$ $9.4, P<0.0001)$, compensating for the $-4.8 \mathrm{D}$ of imposed effective hyperopic defocus difference between the eyes. The eyes wearing the $-5 \mathrm{D}$ lens on average developed $-0.99 \pm 0.69$ $\mathrm{D}$ of myopia, while the fellow eyes remained relatively hyperopic $(+3.86 \pm 0.35 \mathrm{D})$, the latter expected for young animals of this age as complete emmetropization takes approximately 30 days. ${ }^{30}$

In experiment 1, the average relative myopia induced by 7 days of $-5 \mathrm{D}$ lens wear was $-5.35 \pm 0.78 \mathrm{D}(N=9)$. The biometric data for each subset of animals in each experiment are shown in Table 2 . In the subset of animals in experiment 1 randomly assigned to the myopia group, the lens-wearing eyes became myopic in every animal (Fig. 1A; Table 2) and the relative myopia was high $\left(-7.39 \pm 0.73 \mathrm{D}, t_{4}=10.4, P<\right.$ $0.001)$. In the myopia recovery group, the relative myopia before lens removal was $-3.76 \pm 0.17 \mathrm{D}$ (Table 2), which regressed during the 3 days of recovery in each animal (to an average difference between the fellow and recovered eye of $-0.53 \pm 1.15 \mathrm{D}$, Fig. 1B). The average relative recovery calculated across individual animals (change in $-5 \mathrm{D}$ eye minus the change in the fellow eye) was a gain of $+2.90 \pm 1.07 \mathrm{D}$ of hyperopia. If the change in refractive error was linear over these short time periods (which is consistent with our unpublished observations), then the myopia group developed -1.06 diopters per day of relative myopia, while the recovery group developed +0.97 diopters per day of relative hyperopia. Normal untreated animals remained hyperopic (Table 2) and their refractive error did not differ either between the two eyes $\left(t_{4}=-0.53, P=0.63, N=5\right.$ ) or relative to fellow contralateral control eyes (Fig. 1).

In experiment 2 , animals developed $-4.19 \pm 0.62 \mathrm{D}$ of relative myopia after 7 days of $-5 \mathrm{D}$ lens wear $\left(t_{10}=6.8, P<\right.$ 0.001 , Fig. 2A) and a significant increase in ocular length (difference between the eyes of $147 \pm 48 \mu \mathrm{m}, t_{10}=3.1, P<$ 0.001 , Fig. 2B). These changes were accompanied by a significant increase in the depths of the anterior chamber and the vitreous chamber, and a slightly thicker lens relative to fellow contralateral control eyes (difference between the eyes of $85 \pm 36 \mu \mathrm{m}, P=0.042,45 \pm 12 \mu \mathrm{m}, P=0.005$, and $24 \pm 11$ $\mu \mathrm{m}, P=0.047$, respectively). The difference in refractive error was correlated with the difference in ocular length $\left(r^{2}=0.82\right.$, 
A

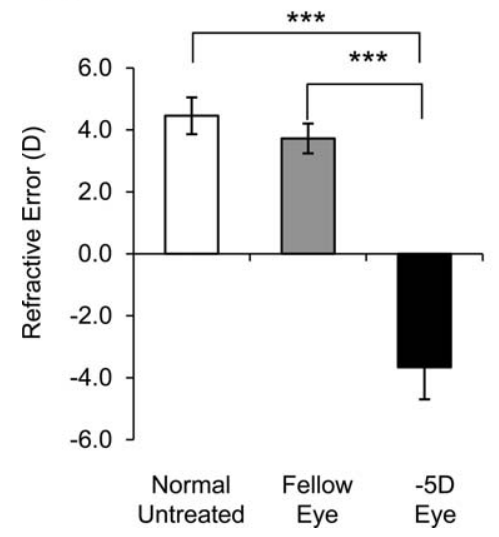

B

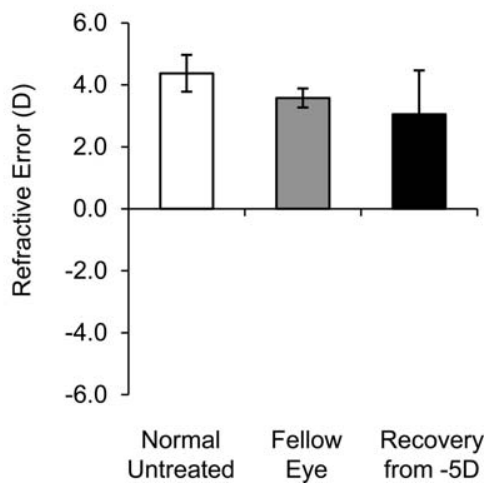

Figure 1. Mean refractive error (mean spherical equivalent) from eyes analyzed for Egr-1 mRNA expression in experiment 1. (A) Mean refractive error in $-5 \mathrm{D}$ monocular lens-wearing eyes and fellow contralateral control eyes after 7 days of treatment and in normal eyes in untreated animals from the same litters as experimental animals $\left(F_{2,14}=36.1, P<0.001\right)$. (B) Mean refractive error in eyes 3 days after removal of the $-5 \mathrm{D}$ monocular lens, following 7 days of lens treatment, compared to that in fellow contralateral control eyes and litter-matched normal untreated eyes $\left(F_{2,11}=0.6\right.$, $P=0.58)$. Holm-Sidak comparisons after one-way ANOVA are shown where ${ }^{* * * *} P<0.001$.

$\left.F_{1,10}=41.3, P<0.001\right)$. On average, eyes developed $-1 \mathrm{D}$ of refractive change for every $30 \mu \mathrm{m}$ in elongation, which is consistent with that predicted from the model eye for the guinea pig. ${ }^{30}$

\section{Changes in Egr-1 Expression During the 24-Hour Light/Dark Cycle}

Retinal Egr-1 mRNA levels showed a statistically significant variation over the light/dark cycle $\left(F_{5,32}=7.7, P<0.01\right.$, Fig. 3 ). This variation was due to elevated levels in the middle of the day and reduced levels in the middle of the night (see Fig. 3 ). This suggests that Egr-1 expression in the guinea pig retina may have a diurnal and/or circadian rhythm. Therefore, for consistency, all samples for the following experiments were collected at 4 PM, 6 hours after lights were switched on.

\section{Retinal Egr-1 Expression in Response to Minus Lens Wear and Recovery From Myopia}

Seven days of negative lens wear $(-5 \mathrm{D})$ significantly reduced retinal Egr-1 mRNA levels as compared to levels observed in
A

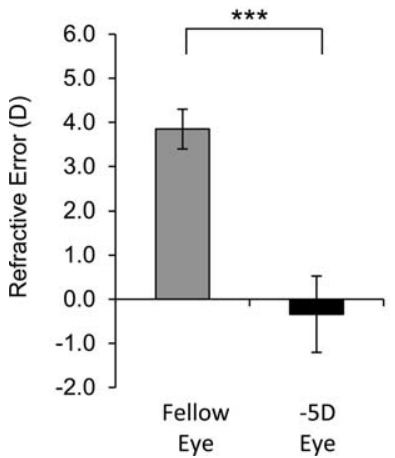

B

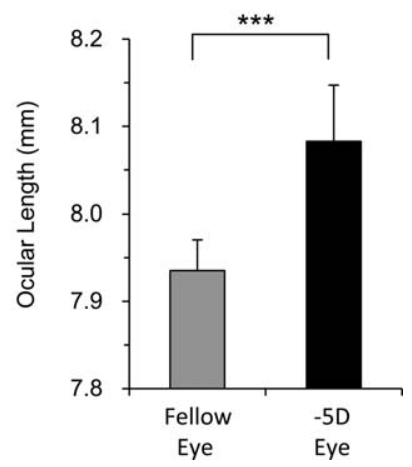

Figure 2. Ocular changes in animals wearing a $-5 \mathrm{D}$ lens on one eye for 7 days in experiment 2. (A) Mean refractive error (mean spherical equivalent) in the lens-wearing eyes and fellow contralateral control eyes. (B) Ocular length (from ultrasonography) in the lens-wearing eyes and fellow contralateral control eyes. ${ }^{* * *} P<0.001$ from matched pair's $t$-test. fellow contralateral control eyes $(0.48 \pm 0.06$ vs. $1.01 \pm 0.25$ MNE, respectively; $51 \% \pm 10 \%$ reduction; Holm-Sidak $t=3.7 ; P$ $=0.019)$ and those observed in age-matched untreated control eyes $(0.48 \pm 0.06$ vs. $1.00 \pm 0.18 \mathrm{MNE}$, respectively; $47 \% \pm$ $6 \%$ reduction; Holm-Sidak $t=3.6 ; P=0.015$; Fig. 4A). Following 7 days of negative lens wear, the spectacle lens was removed to initiate recovery. Three days after lens removal, a significant upregulation in retinal Egr-1 mRNA levels occurred in the recovering eye relative to that seen in the fellow contralateral control eyes $(2.13 \pm 0.16$ vs. $1.06 \pm 0.16 \mathrm{MNE}$, respectively; $212 \% \pm 28 \%$ increase; Holm-Sidak $t=8.8 ; P<0.001$; Fig. $4 \mathrm{~B})$ and that observed in age-matched untreated control eyes $(2.13$ \pm 0.16 vs. $1.00 \pm 0.21 \mathrm{MNE}$, respectively; $234 \% \pm 39 \%$ increase; Holm-Sidak $t=9.3 ; P<0.001$; Fig. 3B).

\section{Egr-1 Immunoreactivity in Untreated Guinea Pig Retinas}

Intense labeling for Egr-1 occurred in cell somas located in the inner nuclear layer (INL; Figs. 5A, 5B, 6B) and the ganglion cell layer (GCL; Figs. 5B, 6A, 6B). The labeled cells in the INL had somas approximately 6 to $8 \mu \mathrm{m}$ in diameter, while those in the GCL were larger $(>10 \mu \mathrm{m})$ (see Fig. 6B for typical comparisons). Light labeling for Egr-1 was also observed within processes that extended vertically through the retina from

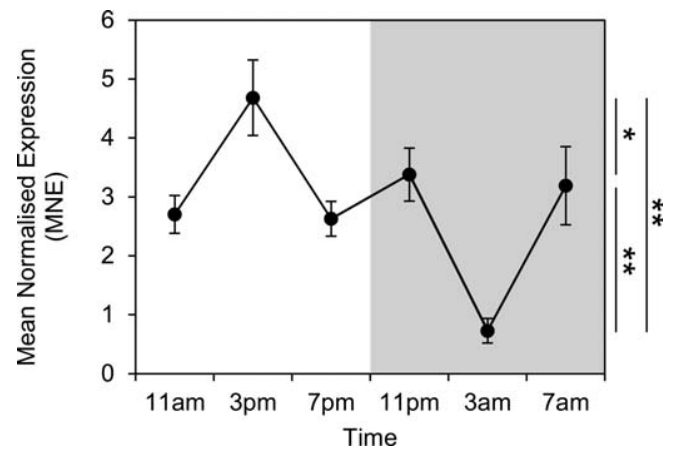

FIgURE 3. Retinal Egr-1 mRNA levels at 4-hour intervals during a 24hour light/dark cycle in untreated animals. Gray area indicates the night period. $N=6$ per time point. $E g r-1 \mathrm{mRNA}$ levels were lower in the middle of the night than the middle of the day. Holm-Sidak comparisons are shown where ${ }^{* * * * *} P<0.001,{ }^{* * * *} P<0.001,{ }^{*} P<0.05$. 
A

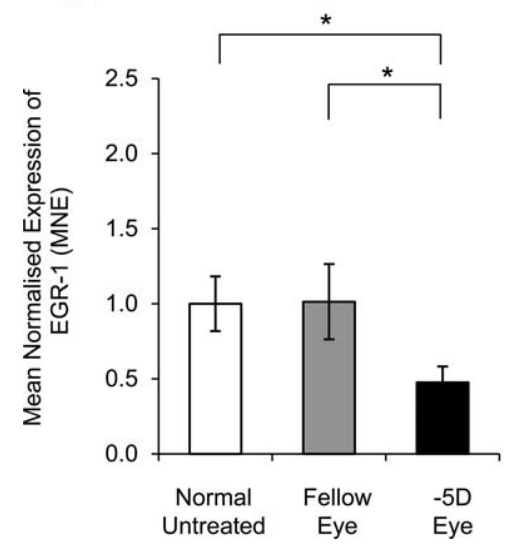

B

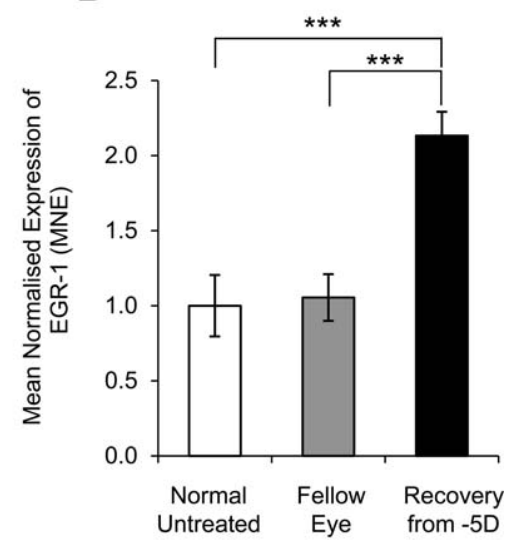

Figure 4. Egr-1 mRNA levels in -5 D lens-wearing eyes compared to fellow contralateral control and litter-matched normal untreated eyes. (A) Egr1 mRNA levels in myopic eyes induced by 7 days of $-5 \mathrm{D}$ lens wear $\left(F_{2,14}=8.7, P=0.01\right)$. (B) Egr-1 mRNA levels during recovery from myopia 3 days after lens removal, following 7 days of $-5 \mathrm{D}$ lens wear $\left(F_{2,11}=55.0, P<0.001\right)$. Holm-Sidak comparisons are shown where ${ }^{*} P<0.05$, ${ }^{* * * *} P<$ 0.001 .

the photoreceptor (PR) layer to the GCL. These processes were most visible within the inner retina (Fig. 5B, horizontal arrows).

Immunoreactivity to PKC $(\alpha, \beta, \gamma)$ was also present in these long processes (Figs. 5C, 5D), which double-labeled for both PKC and Egr-1 (Fig. 5F, horizontal arrows). PKC $(\alpha, \beta, \gamma)$ immunoreactivity could be traced from these processes into end-foot-like structures in the GCL (Figs. 5C, 5D), consistent with the morphology of Müller cells. PKC $(\alpha, \beta, \gamma)$ immunoreactivity was also present in the periphery of the somas of small cells in the INL and their processes. These small PKClabeled cells were located in the bipolar cell layer, and their morphology was consistent with bipolar cells (Figs. 5C, 5D; red arrows). It has previously been observed that PKC- $\alpha$ labels rod ON-bipolar cells in many species, including guinea pigs. ${ }^{34-36}$ The globular terminals of these bipolar-like cells were also intensely labeled for PKC $(\alpha, \beta, \gamma)$ (Figs. 5C, 5D), consistent with axon terminals of rod ON-bipolar cells. ${ }^{34}$ We did not observe Egr-1 labeling of either the somas or terminals of these PKC-labeled small cells (Figs. 5E, 5F). Their terminals were located in the ON strata of the inner plexiform layer (IPL), close to cell somas in the GCL (Fig. 5I). Some, but not all, of these latter somas in the GCL (approximately 20-25 cells per retinal section) were also IR for Egr-1 (Figs. 5F, 5J, 5K; circled).

Gamma-aminobutyric acid immunoreactivity was detected in cell somas in the inner sublayer of the INL where amacrine cell bodies are found and in the periphery of somas in the GCL (Figs. 6C, 6D). Amacrine cells that labeled for Egr-1, but not for GABA, were also observed within the INL (see example in Fig. 6F). However, some of the cells that labeled for Egr-1 in the GCL (Figs. 6A, 6B) also double-labeled for GABA on the edge of their somas, although not all did (Figs. 6E, 6F). In doublelabeled sections, approximately half of the somas in the GCL that labeled for Egr-1 also labeled for GABA. Incubation with mouse (Fig. 5G) or rabbit (Fig. 5H) secondary antibodies only (negative controls) showed weak autofluorescence and no cell labelling.

\section{Sequencing of Egr-1 mRNA PCR Fragments From Guinea Pig Retina}

Sequence analysis of the PCR fragments obtained from guinea pig retinal samples showed an expected fragment size of 180 bp (Fig. 7). The guinea pig Egr-1 mRNA fragment showed significant homology to its closest phylogenetic relatives in the rodent family, namely, the rat (86\%) and mouse (85\%) (Fig. 7). Surprisingly, the guinea pig sequence showed even greater homology with that of the Primate order, more specifically, humans (89\%), rhesus monkeys (89\%), and chimpanzees (89\%). However, in the guinea pig, there was a 3-bp insert at the 126-bp mark, making this fragment of the Egr-1 mRNA sequence $180 \mathrm{bp}$ long in rodents as compared to $177 \mathrm{bp}$ long in primates. Phylogenetic tree reconstruction from sequence relationships (dendrogram), using Clustal W 2.1, indicated three distinct groupings, namely, Rodentia (less the guinea pig), Primates, and the guinea pig.

\section{Discussion}

In different species studied (chicken, rhesus monkey, and mice), manipulations that increase the rate of ocular growth are associated with a rapid downregulation in Egr-1 mRNA 23-25,27 and/or peptide levels. ${ }^{22,28,37}$ In these studies, measures were made within 30 minutes or hours after the introduction of either a diffuser or a negative lens and in some cases, unexpected changes were found in the contralateral control eye. Changes in Egr-1 mRNA expression are also associated with the onset and offset of light. ${ }^{27}$ However, changes in Egr-1 expression are not just associated with light transients, as longer-term changes have been reported in the chick. ${ }^{23}$ Specifically, when tracking the response to $-10 \mathrm{D}$ lens wear or diffuser wear over several days, it was found that an initial rapid downregulation in Egr-1 RNA levels was sustained throughout the period of increased growth. Furthermore, in mice, downregulation in Egr-1 mRNA levels is much greater after diffuser wear than after an equal light attenuation from wearing neutral density filters. ${ }^{27}$ This suggests that the changes in Egr-1 expression can be long-lasting, and not simply due to a transient change in luminance associated with the application of a lens or diffuser.

In the current study, a two-fold downregulation in Egr-1 mRNA levels occurred at a relatively advanced stage in the response to negative lens wear in a mammalian retina. This is consistent with the findings that changes in Egr-1 immunoreactivity are similar for different defocus exposure periods, whether minutes or hours. ${ }^{22,28,37}$ It is curious that such a robust suppression in the level of $E g r-1$ was seen after 7 days of lens wear. By this time, the eyes had compensated for the imposed defocus, so that little refractive error difference remained between the two eyes (measured with the 

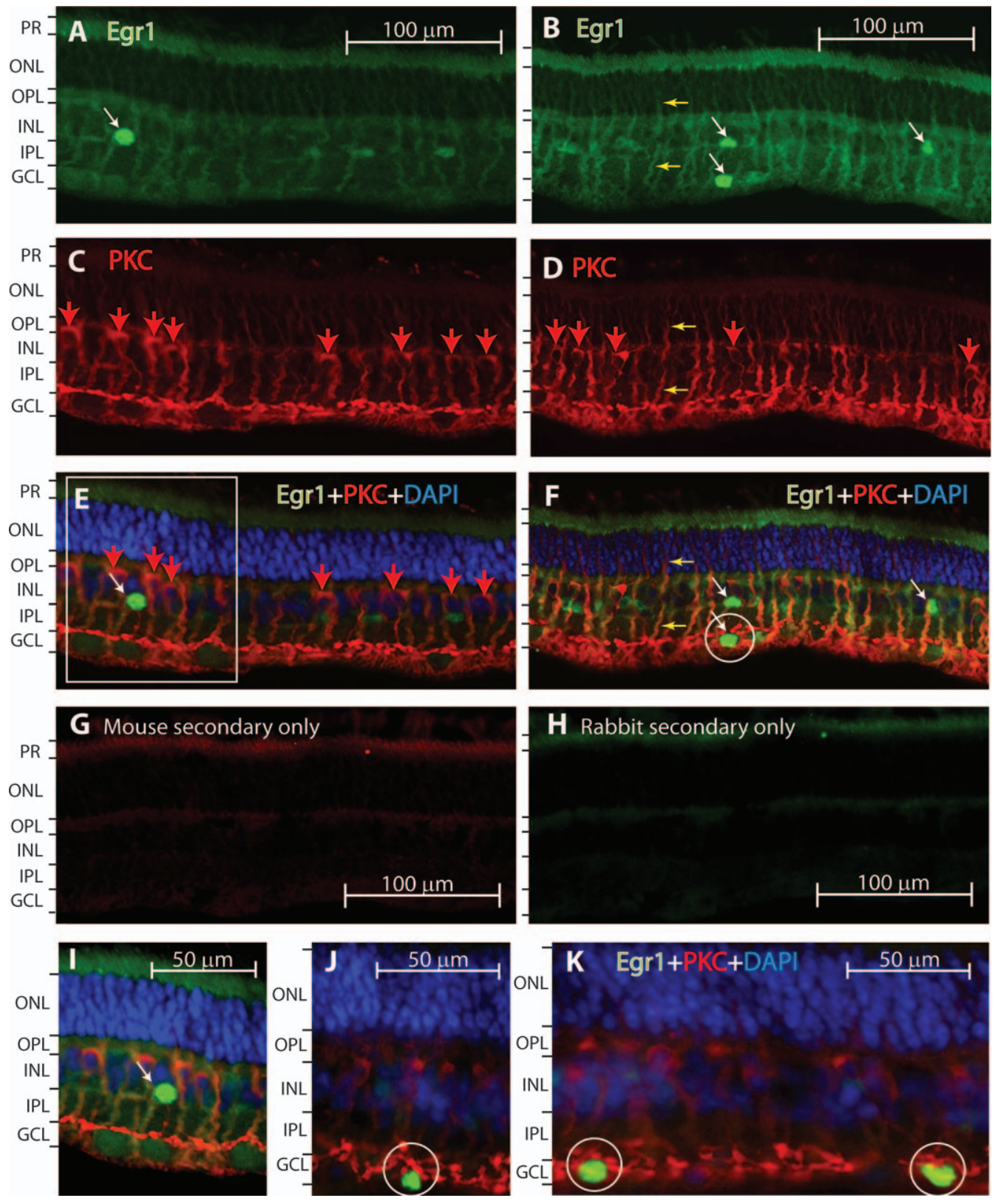

Figure 5. Immunoreactivity in vertical $15-\mu \mathrm{m}$ sections from untreated guinea pig retinas showing Egr-1, PKC, and merged labeling. (A, B) Typical immunoreactivity from Egr-1 antibody labeling (green). Intensely labeled somas for Egr-1 were present in the INL and GCL (white arrows). Light labeling for Egr-1 was also observed in long processes that extended vertically through the retina (one example is indicated by the yellow arrows) $(\mathbf{C}, \mathbf{D})$ Matching sections from (A, B) labeled with PKC $(\alpha, \beta, \gamma)$ (red) showing labeling in bipolar cells (red arrows), in associated axons, and intense labeling within globular terminals in the inner layer of the IPL. Cells similar to Müller cells were also weakly labeled (example processes are indicated by the horizontal yellow arrows). (E, F) Triple-labeled sections for Egr-1, PKC, and DAPI from the same sections shown in (A, C) and (B, D), respectively. The only overlap between PKC and Egr-1 labeling was in the long processes that vertically spanned the retina (borizontal yellow arrows). Close proximity of PKC-containing terminals to Egr-1-labeled somas in the GCL were also observed (see circled example in [F]). Boxed area in (E) is enlarged in (I). (G, H) Incubation with secondary antibodies only (negative controls) showed weak autofluorescence and no cell labeling. (I-K) Merged images triple-labeled for Egr-1 (green), PKC (red), and DAPI (blue), showing proximity of PKC terminals and Egr-1-labeled cells in the GCL. PKC, protein kinase C; DAPI, 4',6-diamidino-2-phenylindole, a nuclear stain that binds strongly to A-T rich regions in DNA. Retinal layers are defined as: PR, photoreceptor; ONL, outer nuclear layer; OPL, outer plexiform layer; INL, inner nuclear layer; IPL, inner plexiform layer; and GCL, ganglion cell layer.

spectacle lens in place). A similar result has been observed in chicks, where Egr-1 mRNA levels do not return to control values until 3 to 4 days after refractive compensation has been achieved to a $-10 \mathrm{D}$ lens. ${ }^{23}$ However, the lack of a refractive error difference does not imply that there is no myopic response in progress. In guinea pigs, part of the myopic response is contributed by changes in corneal power, while the changes in vitreous elongation take approximately 12 days to fully compensate ${ }^{38}$ (Leotta AJ, et al. IOVS 2011;52:ARVO E-Abstract 6302). In chicks, choroidal thinning also rapidly assists in eliminating some of the hyperopic defocus before subsequent changes in eye growth occur. ${ }^{39}$ Therefore, changes in ocular growth may lag behind the initiating defocus stimulus. 

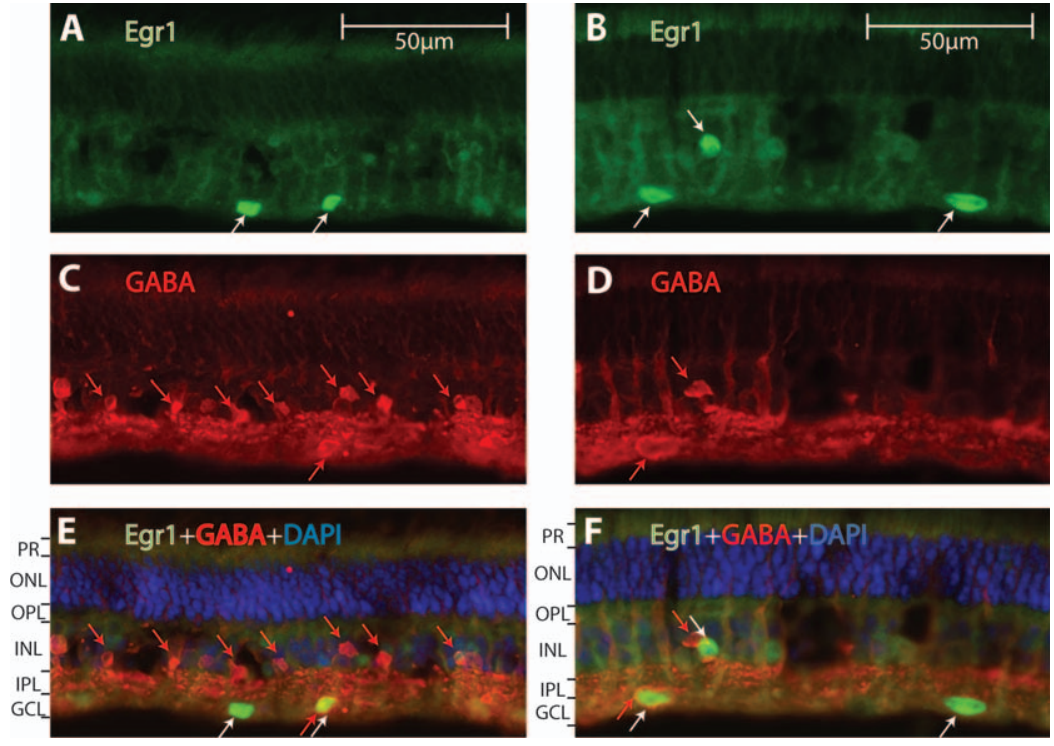

FigurE 6. Immunoreactivity in vertical $15-\mu \mathrm{m}$ sections from untreated guinea pig retinas showing Egr-1, GABA, and merged labeling. (A, B) Retinas labeled with Egr-1 antibody (green) showing typical immunoreactivity in cell bodies within the INL (downward arrow) and GCL (upward arrows). (C, D) Retinas labeled with GABA antibody (red), showing typical immunoreactivity in assumed amacrine cells (downward red arrows) and cells in the GCL (upward red arrows). The latter could be displaced GABAergic amacrine cells. (E, F) Merged sections showing triple-labeling for Egr-1 (green), GABA (red), and DAPI (blue) of the same sections shown in (A, C) and (B, D), respectively. Double-labeling for Egr-1 and GABA was observed in some cells in the GCL (double upward arrows), but not all. Double-labeling was not observed within the amacrine cell sublayer of the INL (see example in $[\mathrm{F}]$ ). See Figure 5 caption for abbreviations.

The driving stimulus behind the Egr-1 downregulation could be either the initial optical defocus or some unidentified myopia-generating retinal growth signal. Interestingly, the induction of genes within the immediate early gene class are not necessarily rapid and transient, but can also be characterized by a delayed and persistent change in expression. ${ }^{21}$ In mice, it has been shown that after controlling for the effect of changes in retinal illumination associated with diffuser wear, Egr-1 mRNA expression is downregulated by $32 \%$ in eyes exposed to only 30 minutes of visual stimulation during form deprivation, presumably a period too short to affect immediate changes in ocular growth. ${ }^{27}$ Taken together, these results suggest that retinal Egr-1 expression is modulated in response to hyperopic defocus, which can lead to a relatively persistent reduction in $E g r-1$ mRNA expression in different species, independent of light-driven transient changes in Egr-1 expression.

When a diffuser or lens is removed after myopia is induced, the eye inhibits its rate of ocular elongation and recovers from
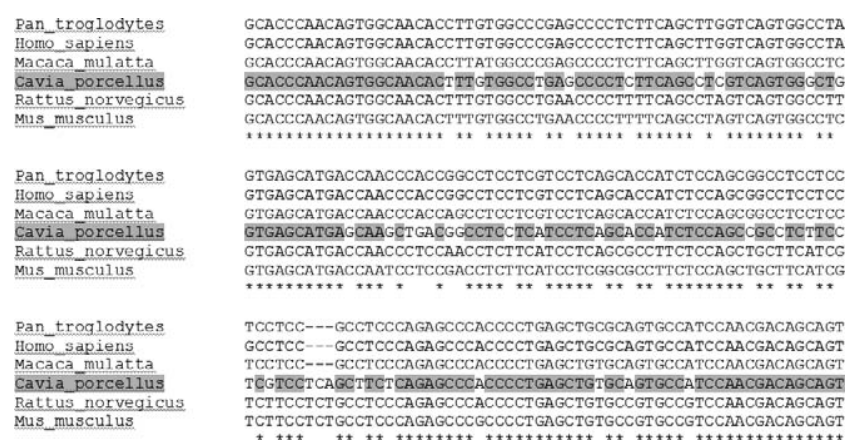

Figure 7. Sequence alignment (Clustal W 2.1) between guinea pig, rat, mouse, human, rhesus monkey, and chimpanzee for the $E g r$-1 gene fragment investigated. Sections highlighted in gray indicate areas of the annotated sequence of the guinea pig Egr-1 fragment that are homologous with the other species. the induced myopia. ${ }^{40}$ In chicks, Egr-1 mRNA levels are rapidly increased during such a period ${ }^{24}$ and return to baseline levels (Ashby RS, et al. IOVS 2007;48:ARVO E-Abstract 5932) once the defocus is fully compensated. ${ }^{41}$ In the current study, we found that Egr-1 mRNA expression doubled in the guinea pig retina after 3 days of recovery from negative lens-induced myopia. In guinea pigs, once a lens is removed after a week of $-5 \mathrm{D}$ monocular lens wear, it takes a further 7 days for the eye to fully recover back to normal. ${ }^{42}$ This recovery process is due to growth inhibition in the enlarged myopic eye, which allows the two eyes to again become equal in length. ${ }^{42}$ In the current study, the relative myopia was $-3.43 \mathrm{D}$ before the recovery period, and 3 days after lens removal, the relative myopia had reduced to $-0.53 \mathrm{D}$, demonstrating that substantial recovery from myopia had taken place. Therefore, the observed upregulation of $E g r-1$ was not confined to the initiation of recovery, but may be sustained throughout the recovery period.

The apparent bidirectional change in the expression of $E g r$ 1 mRNA levels, in response to opposing defocus stimuli in a mammalian eye, suggests that Egr-1 could be a marker associated with the direction of ocular growth. If true, we might also expect to find an upregulation in Egr-1 expression in animals wearing a positive lens, since it is well established that the eye compensates to positive lens wear by inhibiting ocular growth ${ }^{16}$ (see Ref. 42 for review). In agreement with this, an early study has demonstrated that the peptide levels of Egr-1 are elevated in response to 30 minutes and 2 hours of +7 $\mathrm{D}$ lens wear in chicks, although after 30 minutes, the contralateral untreated eyes are also affected. ${ }^{22}$ Similarly, retinal Egr-1 peptide levels appear to be upregulated in the rhesus monkey in response to $+3 \mathrm{D}$ lens wear ${ }^{28}$; however, as explained in the introduction, this may be due to defocus effects in the control eyes wearing plano lenses. A reversal in Egr-1 expression with positive lens wear has not been consistently observed at the RNA level in chicks. Egr-1 mRNA expression in chicks wearing $+7 \mathrm{D}$ lenses for 24 hours is actually reduced by -2 .6-fold (from microarray analysis of 
whole retina), ${ }^{43}$ and -2.1 -fold (based on microarray and PCR analysis of the INL where amacrine cells are located). ${ }^{44}$ This may be due to saturated baseline levels, since tissues were extracted early in the light cycle, and light onset normally causes a significant upregulation in $E g r-1$ expression. ${ }^{27}$ Alternatively, it is possible that the direction of defocus was not as expected in these chick studies, since significant choroidal changes occur within a 24-hour cycle of positive lens wear, ${ }^{39}$ and when the choroid is expanded, the defocus from the positive lens is significantly reduced. ${ }^{45}$ These findings do not argue against the changes observed at the peptide level or the changes we find in eyes recovering from negative lens wear, but do indicate that further work is required to resolve these complexities. It is possible that these differences arise from timing differences, yoking between the eyes, and/or different mechanism(s) for eye growth inhibition in different inhibitory paradigms (McFadden SA, et al. IOVS 2009;50:ARVO E-Abstract 1620).

\section{Diurnal Changes in Egr-1 Expression in the Guinea Pig Retina}

Egr-1 mRNA expression in the guinea pig retina displayed a distinct diurnal cycle, with Egr-1 levels elevated in the middle of the day and reduced in the middle of the night. Similarly, in both chicks and mice, $E g r-1$ mRNA $^{26}$ and peptide ${ }^{22,27}$ levels are observed to be higher during the day than during the night, with a distinct but transient spike in expression seen 0.5 to 1.0 hour following the onset of light, and in some cases, at the cessation of light. ${ }^{27}$ As we did not collect samples within these time windows, we do not know if similar transient spikes in Egr-1 expression also occur in the guinea pig retina. In the current study, we avoided transient light-induced changes in $E g r-1$ expression by consistently extracting retinal tissue in the middle of the day. Similarly, we also avoided any lens-induced light transients (which may occur when a spectacle lens is introduced or removed) by extracting retinas many days after such events occurred.

Since vertebrate eyes normally grow more in the day than at night, ${ }^{46,47}$ Egr-1 levels appear paradoxical, in that they are aligned with normal growth rates (increased when growth is maximal, and decreased when growth is minimal), but inversely related to abnormal growth rates (decreased with accelerated growth, and increased with inhibited growth). A similar dissociation between diurnal growth modulation and abnormal accelerated growth rates has also been observed for dopamine ${ }^{46}$ and glucagon, ${ }^{23,48}$ which have both been postulated to play a role in the suppression of myopic growth. ${ }^{49}$ It is possible that this apparent dissociation arises from a complex interaction related to the phase shift in ocular growth in eyes with accelerated growth, as observed in myopic chick eyes whose axial length rhythm is phase advanced relative to their fellow control eyes. ${ }^{47}$

\section{Immunolocalization of Egr-1}

At the protein level, we found that Egr-1 immunoreactivity was present within the INL and GCL of the guinea pig retina. Cells IR for Egr-1 within the INL were located in the inner margin of this layer and could be either amacrine cells or displaced ganglion cells. Although their soma size was smaller than that typical for displaced ganglion cells in other species, such as rabbit, ${ }^{50}$ there is significant variation between species with regard to the size and distribution of displaced ganglion cells, which have not been characterized in the guinea pig retina. In monkeys, it has been concluded that the activated Egr-1 IR somas in the amacrine layer are amacrine cells, because they also double-label for GABA and GAD65. In the current study, we did not observe double-labeling of Egr-1 and GABA within the inner margin of the INL where amacrine cell somas are normally located.

However, we did find a population of large Egr-1-labeled cells in the GCL that also appeared to double-label for GABA. In the guinea pig retina, GABA has been predominantly observed within amacrine cells and, to a lesser extent, within the GCL. ${ }^{51-53}$ From morphology and double-labeling, GABA expression within the guinea pig GCL appears to be associated with displaced amacrine cells. ${ }^{51-53}$ Since GABA has not been noted to be present in ganglion cells of the guinea pig retina, we tentatively conclude that the subpopulation of large cells in the GCL that showed Egr-1 and GABA double-labeling represent displaced amacrine cells. However, since sparse GABA immunoreactivity has been observed within populations of ganglion cells in both humans ${ }^{54}$ and rats, ${ }^{55}$ further detailed studies are desirable. Similarly, it is unclear whether the cells within the GCL that express Egr-1, but do not express GABA, represent true ganglion cells owing to their large soma size, or a second population of Egr-1 non-GABAergic displaced amacrine cells.

Egr-1 labeling was also observed within long processes that were double-labeled with a nonspecific PKC antibody. These processes could be from bipolar cells and/or from Müller cells, as both have been shown to label with PKC antibodies. In guinea pigs, PKC- $\alpha$ labels rod ON-bipolar cells, ${ }^{34}$ while Müller cells are known to contain PKC in guinea pigs $^{56}$ as well as being associated with a calcium-independent isoform, PKC-

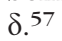

In the monkey retina, defocus-induced changes in Egr-1 expression have also been reported in ON-bipolar cells. ${ }^{27}$ However, in the current study, Egr-1 labeling was not detected in bipolar cell somas. In chicks, modulation of Egr-1 immunoreactivity in lightly labeled PKC- $\alpha$ bipolar cells (cone ON-bipolar cells) is related to changes in light intensity rather than defocus per se. ${ }^{22}$ In the guinea pig retina, we observed PKC-labeled terminals, likely to be from rod ON-bipolar cells. ${ }^{34}$ These terminals appeared in close proximity to cells that exhibited Egr-1 immunoreactivity. However, in the current study, we did not have the resolution to identify synaptic contacts. If direct synaptic contacts were found, suggesting a possible interaction between rod ON-bipolar cells and cells IR for Egr-1 in the GCL, it could implicate the ON pathway in the control of ocular growth. In both monkeys and chicks, Egr-1 immunoreactivity is modulated in ON-bipolar cells in response to defocus and/or light. ${ }^{22,28}$ However, at this stage we can only conclude with certainty that Egr-1 labeling is commonly detected in the retina of different species, and in mammals and primates, may be associated with GABA-expressing neurons.

\section{Gene Sequencing}

Comparison of the 180-bp fragment of the Egr-1 gene amplified from the guinea pig retina with several other species, in which the $E g r$-1 gene has been sequenced, showed surprising results. The guinea pig sequence showed many similarities to its closest phylogenetic relatives in the rodent family, namely, the rat and mouse, such as a specific insert of three base pairs that distinguished rodents from primates. However, the guinea pig sequence also showed many similarities with primates (humans, rhesus monkeys, and chimpanzees), specifically, in regions where there was a distinct difference between members of Primates and those of the Rodentia order. Phylogenetic tree reconstruction based on sequence similarities, using Clustal W 2.1, showed three distinct groupings, namely, Rodentia, Primates, and the guinea pig. This was surprising, as most genes that have been annotated in the 
guinea pig genome appear to be highly conserved between guinea pigs and rats (closest phylogenetic relative that has been sequenced), showing strong sequence homology. A stronger homology with the Rodentia order may be observed once the Egr-1 gene has been completely sequenced for the guinea pig.

\section{The Role of Related Genes in Ocular Growth}

Intravitreal injection of an antisense oligonucleotide for $c$-fos, a member of the IEG family of transcriptional regulators, induces significant myopia in the guinea pig, ${ }^{58}$ while mRNA levels for this IEG are downregulated in the mouse retina after 4 hours of FDM. ${ }^{59}$ These findings suggest that perhaps a variety of Immediate Early Genes (IEGs), such as Egr-1 and $c$-fos, have a central role in the modulation of ocular growth. The genetic locus containing the zinc finger pseudogene $\mathrm{ZC} 3 \mathrm{H} 11 \mathrm{~B}$, a member of the CCCH-type zinc finger family to which Egr-1 belongs, is also strongly associated with axial length in humans. ${ }^{60}$ This latter finding was based on three Genome wide association studies conducted in Chinese and Malay populations. Although the function of this pseudogene is unknown, its coding counterpart (ZC3H11A) has been shown to be downregulated in myopic mice eyes. ${ }^{60}$ Therefore, it is possible that multiple members of the zinc finger family may be involved in the regulatory pathways underlying eye growth. A recent analysis of genomic DNA from leukocytes of 96 high myopes in China has not detected any sequence variations in the Egr-1 gene in this small population. ${ }^{61}$ Taken together with the findings from animal studies, it suggests that any association between Egr-1 activity and the development of myopia in humans is not associated with a loss of function, due to mutation, but rather may be due to a direct change in its expression.

\section{Conclusions}

Egr-1 mRNA levels in a mammalian retina showed a bidirectional persistent response to opposing defocus stimuli, being downregulated after 7 days of negative lens wear (in which hyperopic defocus stimulated growth) and upregulated during recovery following negative lens removal (which suppressed growth in response to myopic defocus). Since Egr-1 is located in the retina, and its expression is a consistent bidirectional marker of ocular growth, it may form an essential part of the growth regulatory pathway(s) across species.

\section{Acknowledgments}

Supported by CG120160, DIISR (Australian Government, CI SAM) and The University of Newcastle. The authors alone are responsible for the content and writing of the paper.

Disclosure: R.S. Ashby, None; G. Zeng, None; A.J. Leotta, None; D.Y. Tse, None; S.A. McFadden, None

\section{References}

1. Wallman J, Turkel J, Trachtman J. Extreme myopia produced by modest change in early visual experience. Science. 1978;201: 1249-1251.

2. Wiesel TN, Raviola E. Myopia and eye enlargement after neonatal lid fusion in monkeys. Nature. 1977;266:66-68.

3. Howlett MH, McFadden SA. Form-deprivation myopia in the guinea pig (Cavia porcellus). Vision Res. 2006;46:267-283.

4. Lodge A, Peto T, McFadden SA. Form deprivation myopia and emmetropization in the guinea pig. Proc Aust Neurosci Soc. 1994;5:17.
5. Shen W, Vijayan M, Sivak JG. Inducing form-deprivation myopia in fish. Invest Ophthalmol Vis Sci. 2005;46:17971803.

6. Smith EL III, Harwerth RS, Crawford ML, von Noorden GK. Observations on the effects of form deprivation on the refractive status of the monkey. Invest Ophthalmol Vis Sci. 1987;28:1236-1245.

7. Wallman J, Adams JI. Developmental aspects of experimental myopia in chicks: susceptibility, recovery and relation to emmetropization. Vision Res. 1987;27:1139-1163.

8. Schaeffel F, Troilo D, Wallman J, Howland HC. Developing eyes that lack accommodation grow to compensate for imposed defocus. Vis Neurosci. 1990;4:177-183.

9. Hung LF, Crawford ML, Smith EL. Spectacle lenses alter eye growth and the refractive status of young monkeys. Nat Med. $1995 ; 1: 761-765$.

10. Wallman J, McFadden S. Monkey eyes grow into focus. Nat Med. 1995;1:737-739.

11. Howlett MH, McFadden SA. Spectacle lens compensation in the pigmented guinea pig. Vision Res. 2009;49:219-227.

12. McFadden SA, Wallman J. Guinea pig eye growth compensates for spectacle lenses. Invest Ophthalmol Vis Sci. 1995; 36:758.

13. Graham B, Judge SJ. The effects of spectacle wear in infancy on eye growth and refractive error in the marmoset (Callithrix jacchus). Vision Res. 1999;39:189-206.

14. Gusek-Schneider GC, Martus P. Stimulus deprivation myopia in human congenital ptosis: a preliminary report of 50 unilateral cases. Strabismus. 2000;8:169-177.

15. Brown NA, Hill AR. Cataract: the relation between myopia and cataract morphology. Br J Ophthalmol. 1987;71:405-414.

16. Metlapally S, McBrien NA. The effect of positive lens defocus on ocular growth and emmetropization in the tree shrew. $J$ Vis. 2008;8(3):1.1-12. doi:10.1167/8.3.1.

17. Wildsoet C. Neural pathways subserving negative lens-induced emmetropization in chicks-insights from selective lesions of the optic nerve and ciliary nerve. Curr Eye Res. 2003;27:371385.

18. Troilo D, Wallman J. The regulation of eye growth and refractive state: an experimental study of emmetropization. Vision Res. 1991;31:1237-1250.

19. Norton TT, Essinger JA, McBrien NA. Lid-suture myopia in tree shrews with retinal ganglion cell blockade. Vis Neurosci. 1994;11:143-153.

20. McBrien NA, Moghaddam HO, Cottriall CL, Leech EM, Cornell LM. The effects of blockade of retinal cell action potentials on ocular growth, emmetropization and form deprivation myopia in young chicks. Vision Res. 1995;35:1141-1152.

21. Caputto BL, Guido ME. Immediate early gene expression within the visual system: light and circadian regulation in the retina and the suprachiasmatic nucleus. Neurochem Res. 2000;25:153-162.

22. Fischer AJ, McGuire JJ, Schaeffel F, Stell WK. Light- and focusdependent expression of the transcription factor ZENK in the chick retina. Nat Neurosci 1999;2:706-712.

23. Ashby R, Kozulin P, Megaw PL, Morgan IG. Alterations in ZENK and glucagon RNA transcript expression during increased ocular growth in chickens. Mol Vis. 2010;16:639-649.

24. Ashby R, McCarthy CS, Maleszka R, Megaw P, Morgan IG. A muscarinic cholinergic antagonist and a dopamine agonist rapidly increase ZENK mRNA expression in the form-deprived chicken retina. Exp Eye Res. 2007;85:15-22.

25. Simon P, Feldkaemper M, Bitzer M, Ohngemach S, Schaeffel F. Early transcriptional changes of retinal and choroidal TGFbeta2, RALDH-2, and ZENK following imposed positive and negative defocus in chickens. Mol Vis. 2004;10:588-597. 
26. Schippert R, Burkhardt E, Feldkaemper M, Schaeffel F. Relative axial myopia in Egr-1 (ZENK) knockout mice. Invest Opbthalmol Vis Sci. 2007;48:11-17.

27. Brand C, Burkhardt E, Schaeffel F, Choi JW, Feldkaemper MP. Regulation of Egr-1, VIP, and Shh mRNA and Egr-1 protein in the mouse retina by light and image quality. Mol Vis. 2005;11: 309-320.

28. Zhong $\mathrm{X}$, Ge J, Smith EL III, Stell WK. Image defocus modulates activity of bipolar and amacrine cells in macaque retina. Invest Ophthalmol Vis Sci. 2004;45:2065-2074.

29. Mathis U, Schaeffel F. Glucagon-related peptides in the mouse retina and the effects of deprivation of form vision. Graefes Arch Clin Exp Ophthalmol. 2007;245:267-275.

30. Howlett MH, McFadden SA. Emmetropization and schematic eye models in developing pigmented guinea pigs. Vision Res. 2007;47:1178-1190.

31. McFadden SA, Howlett MH, Mertz JR. Retinoic acid signals the direction of ocular elongation in the guinea pig eye. Vision Res. 2004;44:643-653.

32. Kucharski R, Maleszka R. Microarray and real-time PCR analyses of gene expression in the honeybee brain following caffeine treatment. J Mol Neurosci. 2005;27:269-276.

33. Simon P. Q-Gene: processing quantitative real-time RT-PCR data. Bioinformatics. 2003;19:1439-1440.

34. Shin JA, Kim IB, Lee HY. Changes in retinal neurons in the guinea pig retina stimulated by strobe lights during development. Neurosci Lett. 2012;531:57-62.

35. Wood JP, McCord RJ, Osborne NN. Retinal protein kinase C. Neurochem Int. 1997;30:119-136.

36. Brecha N, Johnson D, Peichl L, Wassle H. Cholinergic amacrine cells of the rabbit retina contain glutamate decarboxylase and gamma-aminobutyrate immunoreactivity. Proc Natl Acad Sci U $S$ A. 1988;85:6187-6191.

37. Bitzer M, Schaeffel F. Defocus-induced changes in ZENK expression in the chicken retina. Invest Ophthalmol Vis Sci. 2002; $43: 246-252$.

38. Leotta AJ, Bowrey HE, Zeng G, McFadden SA. Temporal properties of the myopic response to defocus in the guinea pig. Ophthalmic Physiol Opt. 2013;33:227-244.

39. Nickla DL, Wallman J. The multifunctional choroid. Prog Retin Eye Res. 2010;29:144-168.

40. Wallman J, Winawer J. Homeostasis of eye growth and the question of myopia. Neuron. 2004;43:447-468.

41. Ashby R. Molecular Control of Eye Growth: A Candidate Gene Approach. Canberra, Australia: Research School of Biology, Australian National University; 2007.

42. McFadden SA, Tse DY, Bowrey HE, et al. Integration of defocus by dual power fresnel lenses inhibits myopia in the mammalian eye. Invest Ophthalmol Vis Sci. 2014;55:908-917.

43. Schippert R, Schaeffel F, Feldkaemper MP. Microarray analysis of retinal gene expression in chicks during imposed myopic defocus. Mol Vis. 2008;14:1589-1599.
44. Ashby RS, Feldkaemper MP. Gene expression within the amacrine cell layer of chicks after myopic and hyperopic defocus. Invest Ophthalmol Vis Sci. 2010;51:3726-3735.

45. Wildsoet C, Wallman J. Choroidal and scleral mechanisms of compensation for spectacle lenses in chicks. Vision Res. 1995; 35:1175-1194.

46. Weiss S, Schaeffel F. Diurnal growth rhythms in the chicken eye: relation to myopia development and retinal dopamine levels. J Comp Physiol A. 1993;172:263-270.

47. Nickla DL. The phase relationships between the diurnal rhythms in axial length and choroidal thickness and the association with ocular growth rate in chicks. J Comp Physiol A Neuroethol Sens Neural Behav Physiol. 2006;192:399-407.

48. Feldkaemper MP, Schaeffel F. Evidence for a potential role of glucagon during eye growth regulation in chicks. Vis Neurosci. 2002;19:755-766.

49. Feldkaemper M, Schaeffel F. An updated view on the role of dopamine in myopia. Exp Eye Res. 2013;114:106-119.

50. Robson JA, Hollander H. Displaced ganglion cells in the rabbit retina. Invest Ophthalmol Vis Sci. 1984;25:1376-1381.

51. Agardh E, Bruun A, Ehinger B, Storm-Mathisen J. GABA immunoreactivity in the retina. Invest Ophthalmol Vis Sci. 1986;27:674-678.

52. Guo C, Hirano AA, Stella SL Jr, Bitzer M, Brecha NC. Guinea pig horizontal cells express GABA, the GABA-synthesizing enzyme GAD 65, and the GABA vesicular transporter. J Comp Neurol. 2010;518:1647-1669.

53. Loeliger M, Rees S. Immunocytochemical development of the guinea pig retina. Exp Eye Res. 2005;80:9-21.

54. Crooks J, Kolb H. Localization of GABA, glycine, glutamate and tyrosine hydroxylase in the human retina. J Comp Neurol. 1992;315:287-302.

55. Versaux-Botteri C, Pochet R, Nguyen-Legros J. Immunohistochemical localization of GABA-containing neurons during postnatal development of the rat retina. Invest Ophthalmol Vis Sci. 1989;30:652-659.

56. Mao JF, Liu SZ, Qin WJ, Xiang Q. Modulation of TGFbeta(2) and dopamine by PKC in retinal Müller cells of guinea pig myopic eye. Int J Ophthalmol. 2011;4:357-360.

57. Osborne NN, Wood J, Groome N. The occurrence of three calcium-independent protein kinase $\mathrm{C}$ subspecies (delta, epsilon and zeta) in retina of different species. Brain Res. 1994;637:156-162.

58. Liu SZ, Wei X, Wang JY, Wu XY, Tan XP. Antisense c-fos oligonucleotides-induced myopia in guinea pigs [in Chinese]. Zhong Nan Da Xue Bao Yi Xue Ban. 2007;32:132-137.

59. Brand C, Schaeffel F, Feldkaemper MP. A microarray analysis of retinal transcripts that are controlled by image contrast in mice. Mol Vis. 2007;13:920-932.

60. Fan Q, Barathi VA, Cheng CY, et al. Genetic variants on chromosome 1q41 influence ocular axial length and high myopia. PLoS Genet. 2012;8:e1002753.

61. Li T, Xiao X, Li S, Xing Y, Guo X, Zhang Q. Evaluation of EGR1 as a candidate gene for high myopia. Mol Vis. 2008;14:1309-1312. 